\title{
Poisson Structures for Geometric Curve Flows in Semi-simple Homogeneous Spaces
}

Gloria Marí Beffa

Department of Mathematics

University of Wisconsin

Madison, Wisconsin 53706

maribeff@math . wisc . edu

http://www.math.wisc.edu/ maribeff
Peter J. Olver ${ }^{\dagger}$

School of Mathematics

University of Minnesota

Minneapolis, MN 55455

olver@math . umn. edu

http://www. math.umn.edu/ olver

\begin{abstract}
We apply the equivariant method of moving frames to investigate the existence of Poisson structures for geometric curve flows in semi-simple homogeneous spaces. We derive explicit compatibility conditions that ensure that a geometric flow induces a Hamiltonian evolution of the associated differential invariants. Our results are illustrated by several examples of geometric interest.
\end{abstract}

\section{Introduction.}

In 1972, Hasimoto, [9], showed how the evolution of curvature and torsion of space curves under the vortex filament flow is governed by the completely integrable nonlinear Schrödinger equation. Since then, a large variety of integrable soliton equations, including all of the most familiar examples (Korteweg-deVries, modified Korteweg-deVries, Sawada-Kotera, etc.), have arisen in connection with invariant geometric curve flows in various Klein geometries, $[\mathbf{1}, \mathbf{2}, \mathbf{3}, \mathbf{1 1}, \mathbf{1 4}, \mathbf{1 9}, \mathbf{2 1}]$. However, the underlying reasons for the surprisingly frequent appearance of integrability remain mysterious.

The basic geometric construction begins with a Lie group $G$ acting on a $m$-dimensional manifold $M$ - typically a homogeneous space. A $G$-invariant evolution equation for curves in $M$ is said to define a geometric curve flow. Given a geometric curve flow, our focus will

$\dagger$ Supported in part by NSF Grant DMS 08-07317.

May 24, 2010 
be on the induced evolution of the differential invariants associated with the group action. In favorable situations, they evolve according to a completely integrable bi-Hamiltonian system, cf. [22]. A complete understanding of this phenomenon remains obscure, and so our more modest aim is investigate the Hamiltonian structure of such differential invariant evolutions. We will apply the powerful equivariant moving frame and invariant variational bicomplex machinery developed in $[\mathbf{6}, \mathbf{1 3}, \mathbf{2 4}]$ to analyze the Poisson reduction, to the evolution of the Maurer-Cartan differential invariants associated with the moving frame, of one of the canonical Poisson structures appearing on the loop space of the dual to the Lie algebra of $G$. The geometric description of the resulting reduced Poisson structure was first described in $[\mathbf{1 6}, \mathbf{1 8}]$.

In this paper, we restrict our attention to curve flows in a homogeneous space $M=$ $G / N$, in which $G$ is a semisimple Lie group and $N \subset G$ a closed subgroup. Modulo certain extra complications, our results can be extended to curves in affine geometry; details of the latter construction will appear elsewhere. Moreover, we will distinguish curves that carry different parametrizations. The case of unparametrized curves (i.e., including the reparametrization pseudo-group) will be the subject of future investigations, building on the fact that the moving frame and invariant variational bicomplex constructions are equally valid in this context.

Example 1.1. A basic example is when $M=\mathbb{R}^{3}$ and $G=\mathrm{E}(3)$ is the Euclidean group of rigid motions. According to $[\mathbf{1 1}, \mathbf{1 9}]$, the most general arc-length preserving Euclidean invariant curve flow takes the form

$$
\frac{\partial C}{\partial t}=J \mathbf{t}+\frac{D_{s} J}{\kappa} \mathbf{n}+K \mathbf{b}
$$

where $\mathbf{t}, \mathbf{n}, \mathbf{b}$ are the unit tangent, normal and binormal forming the classical Euclideaninvariant frame, while $J, K$ are arbitrary Euclidean differential invariants, i.e., functions of curvature $\kappa$, torsion $\tau$, and their derivatives with respect to arc length $s$. The induced flow on the curvature and torsion has the form

$$
\frac{\partial}{\partial t}\left(\begin{array}{c}
\kappa \\
\tau
\end{array}\right)=\mathcal{P}\left(\begin{array}{c}
J \\
K
\end{array}\right)
$$

where

$$
\mathcal{P}=\left(\begin{array}{cc}
-\tau D_{s}-D_{s} \tau & D_{s}^{2} \frac{1}{\kappa} D_{s}-\frac{\tau^{2}}{\kappa} D_{s}+D_{s} \kappa \\
D_{s} \frac{1}{\kappa} D_{s}^{2}-D_{s} \frac{\tau^{2}}{\kappa}+\kappa D_{s} & D_{s}\left(\frac{\tau}{\kappa^{2}} D_{s}+D_{s} \frac{\tau}{\kappa^{2}}\right) D_{s}+\tau D_{s}+D_{s} \tau
\end{array}\right) .
$$

It can be checked directly - although this will follow from our general constructions - that $\mathcal{P}$ is a Poisson operator. Thus, if $J, K$ arise as the variational derivatives of a Hamiltonian functional, then (1.2) forms a Hamiltonian flow.

We begin this article by exhibiting a class of first order Poisson differential operators for Hamiltonian curve flows in a vector space. Every such Poisson structure is associated with the loop space $\mathcal{L} \mathfrak{g}^{*}$ based on the dual to some Lie algebra $\mathfrak{g}$, where it can be viewed as a central extension of the classic Lie-Poisson structure on $\mathfrak{g}^{*},[\mathbf{1 2}, \mathbf{2 2}]$. Since the MaurerCartan differential invariants arising from a moving frame on the homogeneous space $G / N$ 
naturally take their values in the Lie algebra $\mathfrak{g}$, we first translate the Poisson evolution to the loop space $\mathcal{L} \mathfrak{g}$. In section 3 , building on earlier work $[\mathbf{1 6}, \mathbf{1 8}]$, we analyze the Poisson reduction, [20], of such structures to the quotient loop space $\mathcal{L} \mathfrak{g} / \mathcal{L} N$.

Our approach to the geometry of invariant curve flows will be based on the equivariant method of moving frames introduced in [6]. General formulas for the evolution of differential invariants under invariant geometric flows were found in [24], and involve certain invariant differential operators that arise naturally in the invariant variational bicomplex of $[\mathbf{1 3}]$. In section 4 , we establish a remarkable factorization of the invariant linearization operator that governs the Maurer-Cartan invariant flow. The first factor can be identified with the pull back, via the Maurer-Cartan map, of the Lie algebra Poisson operator found in the first section, while the second factor, dubbed the Maurer-Cartan operator, also appears naturally in the context of the invariant variational bicomplex.

In section 5 we describe how Poisson reduction works in the differential invariant setting, and in section 6 we investigate the algebraic description of compatibility conditions found in $[\mathbf{1 8}]$ that are required for the reduction of a Hamiltonian flow on $\mathcal{L} \mathfrak{g}^{*}$ to coincide with the differential invariant evolution induced by a geometric curve flow. We then establish the existence of a suitable reduction of the loop space Poisson structure governing compatible flows. We will need to impose certain restrictions on the structure of the Maurer-Cartan invariants that occur in the examples of interest illustrating our results. Extensions of these methods to more general settings will be the subject of future investigations.

\section{Lie Algebraic Poisson Structures.}

We begin by stating a basic classification theorem for first order Poisson operators of a particular form, that are defined on the loop space of a vector space. Each of these Poisson structures induces an identification of the underlying vector space with the dual to some Lie algebra.

Recall that the loop space of a manifold $M$ is, by definition, the space of smooth maps from the unit circle into $M$, denoted $\mathcal{L} M=\mathrm{C}^{\infty}\left(S^{1}, M\right)$. Suppose $M=\mathbb{R}^{r}$, with coordinates $L=\left(L_{1}, \ldots, L_{r}\right)$. Consider a first order $r \times r$ matrix differential operator $\widehat{\mathcal{P}}=\widehat{\mathcal{P}}[L]$ on the loop space $\mathcal{L} M$, whose entries are of the form

$$
\widehat{\mathcal{P}}_{i j}=b_{i j} D_{x}-\sum_{k=1}^{r} c_{i j}^{k} L_{k}
$$

with $b_{i j}, c_{i j}^{k}$ are constants. An easy computation based on the methods of [22; Section 7.2] leads to the following characterization of when such a differential operator defines a Poisson structure.

Theorem 2.1. The first order matrix differential operator $\widehat{\mathcal{P}}$ with entries $(2.1)$ is Poisson if and only if

- $c_{i j}^{k}$ are the structure constants for an $r$-dimensional Lie algebra $\mathfrak{g}$ relative to a basis $\mathbf{v}_{1}, \ldots, \mathbf{v}_{r}$, and 
- $B=\left(b_{i j}\right)$ is a symmetric $r \times r$ matrix, with

$$
\beta=\mu \odot B \mu=\sum_{i, j=1}^{r} b_{i j} \mu^{i} \odot \mu^{j}
$$

an ad*-invariant symmetric 2 tensor on the Lie algebra dual $\mathfrak{g}^{*}$, in which $\mu=$ $\left(\mu^{1}, \ldots, \mu^{r}\right)$ form the dual basis of $\mathfrak{g}^{*}$, i.e., the Maurer-Cartan forms. a point

We can thus identify $\mathbb{R}^{r} \simeq \mathfrak{g}^{*}$, with $L=\left(L_{1}, \ldots, L_{r}\right)$ representing the coordinates of

$$
L=\sum_{i=1}^{r} L_{i} \mu^{i} \in \mathfrak{g}^{*} .
$$

The ad ${ }^{*}$-invariance of $\beta$ requires that

$$
\sum_{l=1}^{r}\left(c_{i j}^{l} b_{l k}+c_{i k}^{l} b_{l j}\right)=0 \quad \text { for all } \quad i, j, k .
$$

In view of this condition, we will refer to $\beta$ as a cocycle. With this convention, the differential operator (2.1) defines a Poisson structure on the loop space $\mathcal{L} \mathfrak{g}^{*}$. The Poisson operator

$$
\widehat{\mathcal{P}}[L] H=B D_{x} H+\operatorname{ad}_{H}^{*}(L), \quad L \in \mathfrak{g}^{*}, \quad H \in \mathcal{L} \mathfrak{g},
$$

maps $\mathcal{L} \mathfrak{g} \rightarrow \mathcal{L} \mathfrak{g}^{*}$. Note that Theorem 2.1 does not require that the cocycle $\beta$ be of maximal rank, and so the Poisson structure can be degenerate. In particular, if $\beta=0$, then $\widehat{\mathcal{P}}[L]$ reduces to the usual Lie-Poisson structure on $\mathfrak{g}^{*},[\mathbf{2 2}]$.

Remark: When $B$ is nondegenerate, this result is due to Dubrovin and Novikov [4; Theorem 3]; see also the survey paper [5]. They further proved that any nondegenerate first order Poisson operator of "hydrodynamic type" can be transformed into the linear canonical form obtained by adding a constant matrix to (2.1).

If $\mathfrak{g}$ is semi-simple, then $\beta$ will be a multiple of the Killing metric tensor; to avoid degenerating back to the Lie-Poisson structure, we assume the multiple is non-zero. Under this restriction, $B$ is an invertible matrix, and the Poisson structure (2.4) is non-degenerate. From here on, for brevity, we will restrict out attention to the semi-simple case, although many of our results can be extended to affine Lie algebras, and some to completely general Lie algebras. An ongoing project is to determine how far these methods can be pushed.

Let $\widehat{H}[L]$ be a Hamiltonian functional defined on $\mathcal{L} \mathfrak{g}^{*}$. For the Poisson structure defined by (2.4), the associated Hamiltonian flow is

$$
\frac{\partial L}{\partial t}=\widehat{\mathcal{P}}[L] \frac{\delta \widehat{H}}{\delta L}=B D_{x} \delta \widehat{H}+\operatorname{ad}_{\delta \widehat{H}}^{*}(L) .
$$

where, on occasion, we abbreviate the variational derivative as $\delta \widehat{H}=\delta \widehat{H} / \delta L$. In coordinates,

$$
\frac{\partial L_{i}}{\partial t}=\sum_{j=1}^{r}\left(b_{i j} D_{x} \frac{\delta \widehat{H}}{\delta L_{j}}-\sum_{k=1}^{r} c_{i j}^{k} L_{k} \frac{\delta \widehat{H}}{\delta L_{j}}\right) .
$$


Let us introduce dual variables $K=\left(K^{1}, \ldots, K^{r}\right)$, which define coordinates on the Lie algebra $\mathfrak{g}$. The cocycle $(2.2)$ defines an invertible linear map $\widehat{\beta}: \mathfrak{g} \rightarrow \mathfrak{g}^{*}$, given by

$$
L=\widehat{\beta}(K)=B K .
$$

Given a Hamiltonian functional $\widehat{H}[L]$ on $\mathcal{L} \mathfrak{g}^{*}$, let

$$
\widetilde{H}[K]=\widehat{H}[B K]=\beta^{*}(\widehat{H}[L])
$$

be the corresponding Hamiltonian functional on $\mathcal{L} \mathfrak{g}$. Clearly, their variational derivatives are related by

$$
\frac{\delta \widetilde{H}}{\delta K}[K]=B \frac{\delta \widehat{H}}{\delta L}[B K]
$$

We now use the cocycle map $\beta$ to pull back the Hamiltonian system (2.5). Substituting $(2.6,8)$, and then using $(2.3,4)$, we find

$$
\begin{aligned}
\sum_{j=1}^{r} b_{i j} \frac{\partial K^{j}}{\partial t} & =\sum_{j=1}^{r} b_{i j} D_{x} \frac{\delta \widehat{H}}{\delta L_{j}}-\sum_{j, k=1}^{r} c_{i j}^{k} b_{k l} K^{l} \frac{\delta \widehat{H}}{\delta L_{j}} \\
& =\sum_{j=1}^{r} b_{i j} D_{x} \frac{\delta \widehat{H}}{\delta L_{j}}-\sum_{j, k=1}^{r} b_{i k} c_{j l}^{k} K^{l} \frac{\delta \widehat{H}}{\delta L_{j}} .
\end{aligned}
$$

Since $B$ is invertible, using (2.8), the Poisson evolution on $\mathcal{L} \mathfrak{g}$ takes the form

$$
\frac{\partial K}{\partial t}=\widetilde{\mathcal{Q}}[K] B^{-1} \frac{\delta \widehat{H}}{\delta K}=\widetilde{\mathcal{P}}[K] \frac{\delta \widetilde{H}}{\delta K},
$$

where $\widetilde{\mathcal{Q}}[K]=\left(\widetilde{\mathcal{Q}}_{i}^{k}\right)$ is the matrix differential operator with entries

$$
\widetilde{\mathcal{Q}}_{i}^{k}=\delta_{i}^{k} D_{x}-\sum_{j=1}^{r} c_{i j}^{k} K^{j}, \quad i, k=1, \ldots, r,
$$

and $\delta_{i}^{k}$ is the usual Kronecker symbol. Note that

$$
\widetilde{\mathcal{Q}}[K](J)=\left(D_{x}+\operatorname{ad}_{K}\right) J=D_{x} J+[K, J] \quad \text { for any } \quad J \in \mathcal{L} \mathfrak{g},
$$

and satisfies

$$
\widehat{\mathcal{P}}[L]=B \widetilde{\mathcal{Q}}[K] \quad \text { when } \quad L=B K
$$

On the other hand,

$$
\widetilde{\mathcal{P}}[K]=\widetilde{\mathcal{Q}}[K] B^{-1}=B^{-1} \widehat{\mathcal{P}}[B K] B^{-1}
$$

is the pull-back of the Poisson operator (2.4) under the map (2.6). As a result, $\widetilde{\mathcal{P}}$ automatically defines a Poisson structure on $\mathcal{L} \mathfrak{g}$. 


\section{Poisson Reduction.}

Let $G$ be an $r$ dimensional Lie group, acting on a $q=r-s$ dimensional homogeneous space $M=G / N$ for some $s$ dimensional closed subgroup $N \subset G$. The Lie algebras of $N$ and $G$ are denoted, respectively, by $\mathfrak{n} \subset \mathfrak{g}$, while $\mathcal{L} \mathfrak{n} \subset \mathcal{L} \mathfrak{g}$ are the associated loop spaces.

In preparation for our study of Poisson structures on invariants, we investigate the reduction of the Poisson structure (2.14) on $\mathcal{L} \mathfrak{g}$ to the quotient loop space $\pi: \mathcal{L} \mathfrak{g} \rightarrow \mathcal{L} \mathfrak{g} / \mathcal{L} N$. Here $\mathcal{L} N$ acts on $\mathcal{L} \mathfrak{g}$ via the loop extension of the adjoint action

$$
A(N) \cdot K=N^{-1} N_{x}-\operatorname{Ad}_{N}(K) \quad \text { for } \quad N \in \mathcal{L} N .
$$

The infinitesimal action is given by

$$
a(n) \cdot K=n_{x}-\operatorname{ad}_{n}(K)=n_{x}-[n, K]=n_{x}+\operatorname{ad}_{K}(n)=\widetilde{\mathcal{Q}}[K] n \quad \text { for } \quad n \in \mathcal{L} \mathfrak{n} .
$$

These actions are the dual to the actions on $\mathcal{L} \mathfrak{g}^{*}$ described in [12], and induced by a central extension of the algebra of loops. We refer the reader to [20] for the general theory of Poisson reduction, and $[\mathbf{1 6}, \mathbf{1 8}]$ for Poisson reductions related to moving frames and differential invariants.

Let $\widetilde{h}[k]$ be a functional defined on the quotient, so $k \in \mathcal{L} \mathfrak{g} / \mathcal{L} N$. Let $\widetilde{H}[K]=$ $\widetilde{h}[\pi(K)]$ denote the corresponding functional on $\mathcal{L} \mathfrak{g}$, which will be constant along the orbits of $\mathcal{L} N$ under the action (3.1). This, infinitesimally, requires

$$
0=\langle\delta \widetilde{H}, \widetilde{\mathcal{Q}}[K] n\rangle=\left\langle\delta \widetilde{H}, B^{-1} \widehat{\mathcal{P}}[B K] n\right\rangle=-\left\langle\widehat{\mathcal{P}}[B K] B^{-1} \delta \widetilde{H}, n\right\rangle \quad \text { for } \quad n \in \mathfrak{n} .
$$

Thus, $\widehat{\mathcal{P}}[B K] B^{-1} \delta \widetilde{H} \in \mathfrak{n}_{0}^{*}$, where $\mathfrak{n}_{0}^{*}=\mathfrak{n}^{\perp} \subset \mathfrak{g}^{*}$ is the annihilator of $\mathfrak{n}$. (This notation is atypical; other authors use $\mathfrak{n}_{0} \subset \mathfrak{g}^{*}$ to denote the annihilator. Our notation here follows the convention of consistently labeling objects that are associated with the Lie algebra dual with $\mathrm{a}^{*}$.) Equivalently, using (2.13),

$$
\widetilde{\mathcal{Q}}[K] B^{-1} \delta \widetilde{H} \in \mathfrak{n}_{0},
$$

where $\mathfrak{n}_{0}=B^{-1} \mathfrak{n}_{0}^{*} \subset \mathfrak{g}$ is the corresponding subspace of the Lie algebra.

Let $\mathcal{L} \mathfrak{a} \subset \mathcal{L} \mathfrak{g}$ denote a cross-section to the $\mathcal{L} N$ orbits. For simplicity, we will assume from now on that $\mathcal{L} \mathfrak{a}$ is an affine subspace of $\mathcal{L} \mathfrak{g}$ (such is the case in all our examples). This condition is not actually necessary until we arrive to the compatibility condition (see $[\mathbf{1 8}]$ ), but it serves to greatly simplify our arguments.

We can thus identify $\varphi: \mathcal{L} \mathfrak{g} / \mathcal{L} N \simeq \mathcal{L} \mathfrak{a} \subset \mathcal{L} \mathfrak{g}$, whereby $\pi \circ \varphi=\mathbb{1}$. Then the original Hamiltonian $\widetilde{h}[k]$ will coincide with its extension $\widetilde{H}[K]=\widetilde{h}[\pi(K)]$ when restricted to the affine subspace $\mathfrak{a}$ :

$$
\widetilde{H}[\varphi(k)]=\widetilde{h}[k] .
$$

We claim that this implies the existence of a differential operator $\widetilde{\mathcal{R}}[k]$ acting on $\mathcal{L} \mathfrak{g}^{*}$ such that

$$
\frac{\delta \widetilde{H}}{\delta K}[\varphi(k)]=B \widetilde{\mathcal{R}}[k] \frac{\delta \widetilde{h}}{\delta k}[k] .
$$

(The $B$ factor is added for later convenience.) In particular, $\widetilde{\mathcal{R}}$ restricted to the dual subspace $\mathcal{L} \mathfrak{a}^{*} \subset \mathcal{L} \mathfrak{g}^{*}$ is the identity. Under these conditions, the following proposition is valid: 
Proposition 3.1. The differential operator $\widetilde{\mathcal{R}}[k]$ is uniquely determined by the condition

$$
\widetilde{\mathcal{Q}}[\varphi(k)] \widetilde{\mathcal{R}}[k] \delta \widetilde{h}[k] \in \mathfrak{n}_{0}, \quad \text { for all } \quad k \in \mathcal{L} \mathfrak{a} .
$$

Proof: Instead of proving that $\widetilde{\mathcal{R}}$ is uniquely determined, we will prove that $\delta \widetilde{H}[\varphi(k)]$ is uniquely determined by $\delta \widetilde{h}[k]$ and $k$. The existence of $\widetilde{\mathcal{R}}$ will thus follow.

On the one hand, since $\widetilde{H}$ coincides with $\widetilde{h}$ on $\mathcal{L} \mathfrak{a}$, its variational derivative $\delta \widetilde{H} \in \mathcal{L} \mathfrak{g}^{*}$ is determined in the direction of $\mathcal{L} \mathfrak{a}^{*}$. In other words, the operator $\widetilde{\mathcal{R}}$ restricted to a section of $\mathcal{L} \mathfrak{a}^{*}$ is the identity. On the other hand, condition (3.3) also determines the value of $\delta \widetilde{H}$ on $\widetilde{\mathcal{Q}}(\mathcal{L} \mathfrak{n})$ to be zero. But $(3.2)$ implies that $\widetilde{\mathcal{Q}}(\mathcal{L} \mathfrak{n})$ is the space tangent to the orbits of $\mathcal{L} N$ on $\mathcal{L} \mathfrak{g}$. Thus, $\delta H$ is prescribed on a complement to $\mathcal{L} \mathfrak{a}^{*}$, and so completely determined.

Q.E.D.

Once $\widetilde{\mathcal{R}}$ has been determined, the reduced Poisson bracket evaluated at $\varphi(k) \in \mathcal{L} \mathfrak{a}$ is described by

$$
\widetilde{\mathcal{P}}_{R}[k]=\widetilde{\mathcal{R}}^{*}[k] \widetilde{\mathcal{Q}}[\varphi(k)] \widetilde{\mathcal{R}}[k]=\widetilde{\mathcal{R}}^{*}[k] \widehat{\mathcal{P}}[B \varphi(k)] \widetilde{\mathcal{R}}[k] .
$$

This satisfies the conditions to be a Poisson operator on $\mathcal{L} \mathfrak{a} \simeq \mathcal{L} \mathfrak{g} / \mathcal{L} N$ as a consequence of the general theory in $[\mathbf{2 0}]$, which relies on the fact that $N$ is a closed subgroup, and so $G / N$ is a smooth manifold.

\section{Moving Frames for Parametrized Curves.}

In this section, we review the equivariant formulation of moving frames, as first exposed in [6], along with relevant parts of the resulting invariant variational bicomplex, [13], in the context of parametrized curves $u: X=\mathbb{R} \rightarrow M=G / N$ in a homogeneous space. Here, "parametrized" means that we do not identify curves that have different underlying parametrizations. We also refer the reader to $[\mathbf{7}, \mathbf{8}]$ for the classical moving frame method in this context.

Let $\mathrm{J}^{n}=\mathrm{J}^{n}(\mathbb{R}, M)$ be the curve jet bundle of order $n$. Let ${ }^{\dagger} \rho: \mathrm{J}^{n} \rightarrow G$ be a left moving frame - that is a left-equivariant map: $\rho\left(g \cdot u^{(n)}\right)=g \cdot \rho\left(u^{(n)}\right)$, where defined. Existence of such a moving frame requires that the order $n$ be sufficiently large in order that $G$ act freely and regularly on an open subset of $\mathrm{J}^{n},[\mathbf{6}]$. The moving frame is uniquely prescribed by the choice of a cross-section $K^{n} \subset \mathrm{J}^{n}$ to the group orbits through the requirement $\rho\left(u^{(n)}\right)^{-1} \cdot u^{(n)} \in K^{n}$ for suitable jets $u^{(n)} \in \mathrm{J}^{n}-$ namely, those belonging to orbits that intersect the cross-section.

Once a moving frame is prescribed, there is an induced invariantization map $\iota$ that takes differential functions $F: \mathrm{J}^{k} \rightarrow \mathbb{R}$ to differential invariants $I=\iota(F)$ and, more generally, differential forms to invariant differential forms. Specifically, given a differential form $\omega$ on $\mathrm{J}^{n}$, its invariantization $\iota(\omega)$ is the unique invariant differential form that agrees on the cross-section: $\iota(\omega)\left|K^{n}=\omega\right| K^{n}$. In particular, invariant forms $\varpi$ are unaffected by invariantization: $\iota(\varpi)=\varpi$. Consequently, invariantization defines an algebra morphism

\footnotetext{
$\dagger$ Our notational conventions allow $\rho$ to only be defined on an open subset of $\mathrm{J}^{n}$.
} 
that projects the exterior algebra of differential forms on $\mathrm{J}^{n}$ to the exterior algebra of invariant differential forms.

We use coordinates $u=\left(u^{1}, \ldots, u^{q}\right)$ on $M=G / N$, so that a parametrized curve has the local coordinate expression $u=u(x)$ depending on the scalar parameter $x \in X=\mathbb{R}$. Let $u_{k}^{\alpha}=D_{x}^{k} u^{\alpha}$ be the induced jet coordinates. We let $I_{k}^{\alpha}=\iota\left(u_{k}^{\alpha}\right)$ be the corresponding differential invariants obtained through the invariantization process. The non-constant combinations of the $I_{k}^{\alpha}$ contain a complete system of functionally independent differential invariants for the prolonged group action.

Since $G$ does not act on the parameter space, the basic invariant horizontal one-form is just

$$
\varpi=\iota(d x)=d x
$$

with $\mathcal{D}=D_{x}$ the dual invariant differential operator that maps differential invariants to differential invariants. Let

$$
\theta_{k}^{\alpha}=d u_{k}^{\alpha}-u_{k+1}^{\alpha} d x, \quad \alpha=1, \ldots, q, \quad k \geq 0
$$

denote the basis contact forms on the curve jet space $\mathrm{J}^{n}$. Let

$$
\vartheta=\left(\vartheta^{1}, \ldots, \vartheta^{q}\right)^{T}=\left(\iota\left(\theta^{1}\right), \ldots, \iota\left(\theta^{q}\right)\right)^{T}
$$

denote the column vector of invariantized zero ${ }^{\text {th }}$ order contact forms.

The induced invariant variational bicomplex, [13], is constructed by decomposing the invariant differential forms into their horizontal and contact components. A differential $k$ form is of type $(i, j)$, for $i+j=k$, if it is a linear combination of terms containing wedge products of $i$ invariant horizontal forms (for curves, $0 \leq i \leq 1$ ) and $j$ invariant contact forms. The invariant variational bicomplex is obtained by splitting $d=d_{\mathcal{H}}+d_{\mathcal{V}}$ into invariant horizontal and vertical components, which relies on the fact that the group acts projectably on the total space $\mathbb{R} \times M$, cf. [13].

While invariantization preserves all algebraic operations among functions and forms, it does not respect differentiation. The universal recurrence formulae, established in [6, 13], relate the derivatives of invariantized objects to the invariantization of their derivatives.

Theorem 4.1. Let $\mathbf{v}_{1}, \ldots, \mathbf{v}_{r}$ be the prolonged infinitesimal generators for the action of $G$ on the curve jet space $\mathrm{J}^{n}$. Let $\iota$ denote the invariantization process associated with a (locally defined) equivariant moving frame $\rho: \mathrm{J}^{n} \rightarrow G$. If $\Omega$ is any differential function or form on $\mathrm{J}^{n}$, then

$$
d \iota(\Omega)=\iota(d \Omega)+\sum_{i=1}^{r} \nu^{i} \wedge \iota\left[\mathbf{v}_{i}(\Omega)\right]
$$

where

$$
\nu=\left(\nu^{1}, \ldots, \nu^{r}\right)^{T}=\left(\rho^{*} \mu^{1}, \ldots, \rho^{*} \mu^{r}\right)^{T}
$$

are the pull-backs of the Maurer-Cartan forms under the moving frame map. 
The pulled-back Maurer-Cartan forms $\nu^{i}$ can, in fact, be determined directly from the recurrence formulae. Suppose that, in local coordinates, the cross-section $K^{n} \subset \mathrm{J}^{n}$ is implicitly defined by the equations

$$
F_{1}\left(x, u^{(n)}\right)=0, \quad \ldots \quad F_{n}\left(x, u^{(n)}\right)=0 .
$$

Then, taking in turn $\Omega=F_{j}, j=1, \ldots, r$, in (4.4) leads to a system of $r$ linear algebraic equations which can be uniquely solved for the $r$ pulled-back Maurer-Cartan forms $\nu^{1}, \ldots, \nu^{r}$. Substituting the results back into (4.4) produces a complete system of recurrence relations for the differential invariants and invariant differential forms.

In particular, the recurrence formulae enable us to explicitly write all the higher order differential invariants $I_{k}^{\alpha}$ for $k \gg 0$ as iterated invariant derivatives of a finite system of generating differential invariants - thus establishing a general Basis Theorem for the algebra of differential invariants, $[\mathbf{6}, \mathbf{2 5}]$. Further, setting $\Omega=\theta_{k}^{\alpha}$ to be a basis contact form, the $(1,1)$ component of $(4.4)$ ends up having the form

$$
\varpi \wedge D_{x}\left(\vartheta_{k}^{\alpha}\right)=\varpi \wedge\left(\vartheta_{k+1}^{\alpha}+\sum_{i=1}^{k-1} \sum_{\beta=1}^{q} J_{i, \beta}^{\alpha} \vartheta_{i}^{\beta}\right),
$$

for certain differential invariants $J_{i, \beta}^{\alpha}$. This allows us to recursively construct formulas expressing the higher order invariant contact forms as linear combinations of invariant derivatives of the order 0 invariant contact forms $\vartheta$ :

$$
\vartheta_{k}^{\alpha}=\iota\left(\theta_{k}^{\alpha}\right)=\mathcal{B}_{k}^{\alpha}(\vartheta)=\sum_{\beta=1}^{q} \mathcal{B}_{k, \beta}^{\alpha}\left(\vartheta^{\beta}\right),
$$

where $\mathcal{B}_{k, \beta}^{\alpha}$ are certain invariant differential operators.

The next step is to split the pulled-back Maurer-Cartan forms into invariant horizontal and invariant contact components, which, in view of (4.6), we write as

$$
\nu=\rho^{*} \mu=\kappa \varpi+\mathcal{C}(\vartheta),
$$

where $\boldsymbol{\kappa}=\left(\kappa^{1}, \ldots, \kappa^{r}\right)^{T}$ are known as the Maurer-Cartan differential invariants, while $\mathcal{C}=\left(\mathcal{C}_{\alpha}^{k}\right)$ is a $r \times q$ matrix of invariant differential operators, which we call the MaurerCartan operator. We can identify the Maurer-Cartan invariants with the components of the Maurer-Cartan map

$$
\boldsymbol{\kappa}\left(u^{(n)}\right)=\rho\left(u^{(n)}\right)^{-1} D_{x} \rho\left(u^{(n)}\right): \mathrm{J}^{n} \longrightarrow \mathfrak{g} .
$$

As noted by Hubert, $[\mathbf{1 0}]$, since the action is transitive on $M$, the Maurer-Cartan invariants $\boldsymbol{\kappa}$ contain a generating set consisting of exactly $q$ independent differential invariants $\varkappa=\left(\varkappa^{1}, \ldots, \varkappa^{q}\right)^{T}$, meaning that every other differential invariant is a function of the $\varkappa$ and their derivatives. Moreover, according to [23], if we employ a moving frame of minimal order, then the generating differential invariants can be taken to be $\varkappa^{\alpha}=\iota\left(u_{k_{\alpha}+1}^{\alpha}\right)$, $\alpha=1, \ldots, q$, where $k_{\alpha}$ is the maximal order derivative of $u^{\alpha}$ that appears in the crosssection normalization equations. In light of the preceding developments, one can construct the explicit formulae for the Maurer-Cartan invariants $\boldsymbol{\kappa}$ and the Maurer-Cartan operator 
$\mathcal{C}$ in terms of the generating invariants using only linear differential algebraic calculations. Examples of these computations can be found below.

Let us now compute the differentials of the pulled-back Maurer-Cartan forms:

$$
d \nu=d \boldsymbol{\kappa} \wedge \varpi+\boldsymbol{\kappa} d \varpi+d[\mathcal{C}(\vartheta)]=d_{\mathcal{V}} \boldsymbol{\kappa} \wedge \varpi+\boldsymbol{\kappa} d \varpi+d[\mathcal{C}(\vartheta)]
$$

since $d_{\mathcal{H}} \boldsymbol{\kappa}=\mathcal{D}(\boldsymbol{\kappa}) \varpi$. We evaluate each of the terms on the right hand side in turn. First,

$$
d \varpi=0,
$$

since $\varpi=d x$ in the parametrized case being considered here. Second, using (4.6),

$$
d_{\mathcal{V}} \boldsymbol{\kappa}=\mathcal{A}(\vartheta)
$$

where $\mathcal{A}=\mathcal{A}_{\kappa}$ is an $r \times q$ matrix of invariant differential operators called the invariant linearization operator associated with the Maurer-Cartan invariants, [24]. Finally, the $(1,1)$ component of the last term of $(4.9)$ is, in view of [13; eq. (5.34)],

$$
d_{\mathcal{H}}[\mathcal{C}(\vartheta)]=\varpi \wedge \mathcal{D}[\mathcal{C}(\vartheta)]
$$

On the other hand, using the moving frame map to pull back the Maurer-Cartan structure equations

$$
d \mu^{i}=-\frac{1}{2} \sum_{j, k=1}^{r} c_{j k}^{i} \mu^{j} \wedge \mu^{k}
$$

we see that the left hand side of (4.9) is given by

$$
d \nu^{i}=-\frac{1}{2} \sum_{j, k=1}^{r} c_{j k}^{i} \nu^{j} \wedge \nu^{k}
$$

where $c_{j k}^{i}$ are the structure constants associated with our choice of basis of $\mathfrak{g}$. Substituting (4.7), the $(1,1)$ component of $(4.14)$ is

$$
-\sum_{j, k=1}^{r} c_{j k}^{i} \kappa^{j} \varpi \wedge \mathcal{C}^{k}(\vartheta)
$$

where $\mathcal{C}^{k}$ denotes the $k^{\text {th }}$ row of the matrix differential operator $\mathcal{C}$.

Plugging equations $(4.10,11,12,15)$ into $(4.9)$ and canceling the common factor $\varpi$, we arrive at the following striking factorization:

$$
\mathcal{A}=\mathcal{Q} \cdot \mathcal{C}
$$

with $\mathcal{C}$ the Maurer-Cartan operator (4.7), while

$$
\mathcal{Q}=\widetilde{\mathcal{Q}}[\boldsymbol{\kappa}] \quad \text { has entries } \quad \mathcal{Q}_{i}^{k}=\delta_{i}^{k} D_{x}-\sum_{j=1}^{r} c_{i j}^{k} \kappa^{j}
$$

Observe that the operator $\widetilde{\mathcal{Q}}$ is obtained by pulling back the Lie algebra operator $(2.12)$ via the Maurer-Cartan map (4.8), i.e., replacing the Lie algebra coordinates $K$ by the Maurer-Cartan invariants $\boldsymbol{\kappa}$. This factorization plays a key role in our subsequent analysis of geometric flows. 


\section{Poisson Brackets Associated to a Moving Frame.}

The moving frame-induced process of replacing coordinates on $\mathfrak{g}$ by the corresponding Maurer-Cartan invariants can be described as a quotient, first established in $[\mathbf{1 5}, \mathbf{1 7}]$ in special cases, and then in [18] for the general semi-simple homogeneous case. This quotient description allows us to reduce the Poisson structure (2.14) on $\mathcal{L} \mathfrak{g}$ to a Poisson structure involving the Maurer-Cartan invariants $\boldsymbol{\kappa}$.

As above, we assume $G$ is a semisimple Lie group and $\pi: G \rightarrow M=G / N$ an associated homogeneous space. We can identify $N=G_{e_{0}}$ as the isotropy subgroup of $e_{0}=\pi(e)$. We will need to assume that the curves $u: \mathbb{R} \rightarrow M$ have monodromy, meaning that there exists $T>0$ such that

$$
u(x+T)=g \cdot u(x), \quad \text { for some } g \in G \text { and for all } x .
$$

The monodromy condition implies that the differential invariants of such a curve are periodic in $x$ with period $T$. We assume that the moving frame $\rho: \mathrm{J}^{n} \rightarrow G$ is based on a cross-section $K^{n} \subset \mathrm{J}^{n}$ sitting over $e_{0}$, that is, $\rho\left(u^{(n)}(x)\right) \cdot e_{0}=u(x)$. A curve (with monodromy) will be called regular if its jet lies in the domain of the moving frame map. The following description can be found in [18].

Lemma 5.1. Under the above assumptions, the image of the Maurer-Cartan map $\kappa: \mathrm{J}^{n} \rightarrow \mathcal{L} \mathfrak{g}$ evaluated on regular curves forms an open subset of the quotient space $\mathcal{L} \mathfrak{g} / \mathcal{L} N \simeq \mathcal{L} \mathfrak{a} \subset \mathcal{L} \mathfrak{g}$.

The identification of Maurer-Cartan invariants is based on the following observations. First, let $u$ be a generic curve in $M \rightarrow G / N$, and let $\rho$ be a left moving frame whose defining cross-section sits over $e_{0}$. Given a set of Maurer-Cartan invariants $\boldsymbol{\kappa}\left(u^{(n)}\right)$, let $K_{\epsilon}(x) \in \mathcal{L} \mathfrak{g}$ be a nearby loop. We can integrate $K_{\epsilon}$ to the group by locally solving the system of ordinary differential equations

$$
g_{\epsilon}^{-1} D_{x} g_{\epsilon}=K_{\epsilon},
$$

producing a curve $g_{\epsilon}(x)$ in $G$. Let $u_{\epsilon}(x)=g_{\epsilon}(x) \cdot e_{0}$ be the corresponding curve in $M$, which clearly satisfies the monodromy condition (5.1). Then

$$
g_{\epsilon}(x)^{-1} \cdot \rho\left(u_{\epsilon}^{(n)}(x)\right) \in \mathcal{L} \mathfrak{n} .
$$

Now, the action (3.1) is induced by the action $g \mapsto g \cdot n$ of $\mathcal{L} N$ on solutions of (5.2). Thus, the algebra element representing Maurer-Cartan invariants for $u_{\epsilon}$ lies in the same $\mathcal{L} N$-orbit as $K_{\epsilon}$.

Keeping Lemma 5.1 and (4.17) in mind, we pull back the operators appearing in Section 3 using the Maurer-Cartan map (4.8) by replacing the coordinates on $\mathfrak{g}$ by the Maurer-Cartan invariants: $K=\boldsymbol{\kappa}\left(u^{(n)}\right)$. Similarly, the coordinates on $\mathcal{L} \mathfrak{g} / \mathcal{L} N \simeq \mathcal{L} \mathfrak{a}$ are replaced by the generating differential invariants: $k=\varkappa\left(u^{(n)}\right)$, with $\kappa=\varphi(\varkappa)$. The resulting operators will be denoted by the same symbols without tildes, so $\mathcal{Q}=\widetilde{\mathcal{Q}}[\boldsymbol{\kappa}]=$ $\widetilde{\mathcal{Q}}[\varphi(\varkappa)]$ and so on. In addition, the pulled back Poisson operator is

$$
\mathcal{P}=\widetilde{\mathcal{P}}[\boldsymbol{\kappa}]=\widetilde{\mathcal{Q}}[\boldsymbol{\kappa}] B^{-1}, \quad \text { where } \quad \boldsymbol{\kappa}=\varphi(\varkappa) .
$$


This operator is skew adjoint, but, without further reduction, is not of the correct shape to be a Poisson operator on the space of differential invariants.

Indeed, the reduced Poisson operator $\mathcal{P}_{R}$ can be found explicitly following two steps: we first apply the Maurer-Cartan invariant version of (3.6) to construct the operator $\mathcal{R}$ :

$$
\mathcal{Q} \mathcal{R} \delta \widetilde{h} \in \mathfrak{n}_{0} .
$$

The reduced Poisson operator is then described by the Maurer-Cartan pull-back of our earlier reduction formula (3.7):

$$
\mathcal{P}_{R}=\mathcal{R}^{*} \mathcal{P} \mathcal{R}
$$

\section{Invariant Curve Flows and Reduced Hamiltonian Systems.}

In general, an invariant curve flow on $M$ takes the form

$$
\frac{\partial C}{\partial t}=J \cdot \mathbf{n}=\sum_{\alpha=1}^{q} J^{\alpha} \mathbf{n}_{\alpha}
$$

where $J=\left(J^{1}, \ldots, J^{q}\right)$ is a vector of differential invariants, and $\mathbf{n}_{1}, \ldots, \mathbf{n}_{q}$ are the groupinvariant normal directions on the curve that are dual to the order zero invariant contact forms (4.3):

$$
\left\langle\vartheta^{\alpha} ; \mathbf{n}_{\beta}\right\rangle=\delta_{\beta}^{\alpha}, \quad \alpha, \beta=1, \ldots, q .
$$

According to [24] and (4.16), the induced evolution of the Maurer-Cartan invariants is then given by

$$
\frac{\partial \kappa}{\partial t}=\mathcal{A}(J)=\mathcal{Q} \mathcal{C}(J)
$$

where $\mathcal{A}=\mathcal{A}_{\kappa}$ is the associated invariant linearization operator (4.11). We will call (6.3) the Maurer-Cartan flow induced by the invariant curve flow (6.1). The Maurer-Cartan flow can clearly be reduced to a flow involving only the generating invariants $\varkappa$.

Our goal is to characterize those geometric flows on $M$ that produce a Hamiltonian flow on the generating differential invariants under the reduced Poisson structure defined by (5.5). Using a direct approach, we can choose a Hamiltonian functional $\widetilde{H}[K]$ that induces the Poisson flow (2.10) on $\mathcal{L} \mathfrak{g}$. Identifying $K=\boldsymbol{\kappa}\left(u^{(n)}\right)$, the corresponding evolution of Maurer-Cartan invariants is

$$
\frac{\partial \boldsymbol{\kappa}}{\partial t}=\mathcal{P}[\boldsymbol{\kappa}] \frac{\delta H}{\delta K}[\boldsymbol{\kappa}]=\mathcal{Q}[\boldsymbol{\kappa}] B^{-1} \frac{\delta H}{\delta K}[\boldsymbol{\kappa}] .
$$

This coincides with the Maurer-Cartan flow (6.3) provided

$$
\mathcal{A}(J)=\mathcal{Q} \mathcal{C}(J)=\mathcal{Q} B^{-1} \frac{\delta H}{\delta K} .
$$

In particular, this holds if $\delta H / \delta K=B \mathcal{C}(J)$. However, examples show that this condition is too restrictive, and a more intricate analysis is required.

We can apply the results of Section 3 to conclude that the Poisson structure on $\mathcal{L} \mathfrak{g}$ defined by (2.14) produces a reduced Poisson bracket on the generating differential 
invariants $\varkappa$. To simplify the construction, we will make the additional assumption that the generating invariants $\varkappa$ occur linearly and algebraically in the full set of MaurerCartan invariants $\boldsymbol{\kappa}$; in other words, we can write $\boldsymbol{\kappa}=A \varkappa+b$ for some constant $r \times q$ matrix $A$ and constant vector $b \in \mathbb{R}^{q}$. This condition depends on an appropriate choice of cross-section. In simple examples, this can always be arranged, but, so far, we do not know general conditions on the group action that guarantee such a cross-section exists. Under this assumption, the Maurer-Cartan map (4.8) traces out an open subset of the affine subspace

$$
\mathfrak{a}=\left\{\boldsymbol{\kappa}=\varphi(\varkappa)=A \varkappa+b \mid \varkappa \in \mathbb{R}^{q}\right\} \subset \mathfrak{g} .
$$

We use this subspace to effect the identification $\mathcal{L} \mathfrak{a} \simeq \mathcal{L} \mathfrak{g} / \mathcal{L} N$.

Remark: The main result that allows us to relate reduced Hamiltonian evolutions on the affine subspace $\mathcal{L} \mathfrak{a}$ traced out by the Maurer-Cartan invariants directly to invariant curve flows was proved in [18] using a somewhat different approach. Notice that the curve evolution in $[\mathbf{1 8}]$ can be written as

$$
\frac{\partial C}{\partial t}=\frac{\partial \Phi}{\partial u}\left(\rho\left(u^{(n)}\right), u\right) J
$$

where $\Phi(g, u)=g \cdot u$ denotes the group action, while $\partial \Phi / \partial u$ is regarded as a linear transformation. On the other hand, the invariantized contact frame (4.2) can also be written as $\vartheta=\frac{\partial \Phi}{\partial u}\left(\rho\left(u^{(n)}\right), u\right) \theta$ and so the flows (6.7) and (6.1) coincide.

Theorem 6.1. Under the above assumptions, the Hamiltonian flow

$$
\frac{\partial \varkappa}{\partial t}=\mathcal{P}_{R}[\varkappa] \frac{\delta h}{\delta \varkappa}
$$

on $\mathcal{L} \mathfrak{a}$ with Hamiltonian functional $h[\varkappa]$ for the reduced Poisson structure (5.5) is induced by the Maurer-Cartan flow (6.3) provided

$$
\mathcal{C}(J) \equiv \mathcal{R} \delta h \bmod \mathfrak{n} .
$$

We call condition (6.9) the compatibility condition. It requires that $\mathcal{C}(J)=\mathcal{R} \delta h+n$ for some $n \in \mathfrak{n}$. We remark that the left hand side, when computed modulo $\mathfrak{n}$, just amounts to an invertible matrix applied to $J$, and hence the compatibility condition uniquely determines the curve flow invariants $J$ in terms of $\delta h$. On the other hand, $\mathcal{R}$ is typically not invertible, and hence only special curve flows are of Hamiltonian form.

Let us investigate how all this works in some basic examples.

Example 6.2. Let $G=\operatorname{PSL}(2)$ be the projective group acting on $M=\mathbb{R}$. The vector fields

$$
\mathbf{v}_{1}=\partial_{u}, \quad \mathbf{v}_{2}=u \partial_{u}, \quad \mathbf{v}_{3}=u^{2} \partial_{u},
$$

serve as a basis for the Lie algebra $\mathfrak{g}=\mathfrak{s l}(2)$. Using the commutation relations, we find that every $\mathrm{ad}^{*}$-invariant symmetric 2 tensor is a scalar multiple of the Killing form:

$$
\beta=\frac{1}{2}\left(\mu^{2}\right)^{2}-2 \mu^{1} \mu^{3}, \quad \text { so that } \quad B=\left(\begin{array}{ccc}
0 & 0 & -1 \\
0 & \frac{1}{2} & 0 \\
-1 & 0 & 0
\end{array}\right) \text {. }
$$


Thus, the cocycle relation (2.6) requires that

$$
L_{1}=-K^{3}, \quad L_{2}=\frac{1}{2} K^{2}, \quad L_{3}=-K^{1} .
$$

The Poisson operator on $\mathcal{L} \mathfrak{g}^{*}$ is

$$
\widehat{\mathcal{P}}[L]=\left(\begin{array}{ccc}
0 & 0 & -1 \\
0 & \frac{1}{2} & 0 \\
-1 & 0 & 0
\end{array}\right) D_{x}+\left(\begin{array}{ccc}
0 & -L_{1} & -2 L_{2} \\
L_{1} & 0 & -L_{1} \\
2 L_{2} & L_{3} & 0
\end{array}\right),
$$

and hence

$$
\widetilde{\mathcal{Q}}[K]=B^{-1} \widehat{\mathcal{P}}[B K]=\mathcal{D}+\left(\begin{array}{ccc}
-K^{2} & K^{1} & 0 \\
-2 K^{3} & 0 & 2 K^{1} \\
0 & -K^{3} & K^{2}
\end{array}\right) .
$$
the form

The prolonged action of $g=\left(\begin{array}{ll}\alpha & \beta \\ \gamma & \delta\end{array}\right) \in \mathrm{SL}(2)$, with $\alpha \delta-\beta \gamma=1$, on curve jets has

$$
\begin{gathered}
v=\frac{\alpha u+\beta}{\gamma u+\delta}, \quad v_{x}=\frac{u_{x}}{(\gamma u+\delta)^{2}}, \quad v_{x x}=\frac{(\gamma u+\delta) u_{x x}-\gamma u_{x}^{2}}{(\gamma u+\delta)^{3}}, \\
v_{x x x}=\frac{(\gamma u+\delta)^{2} u_{x x x}-6 \gamma u_{x} u_{x x}+6 \gamma^{2} u_{x}^{3}}{(\gamma u+\delta)^{4}}, \quad \ldots
\end{gathered}
$$

We use the standard cross-section

$$
K^{2}=\left\{u=0, u_{x}=1, u_{x x}=0\right\}
$$

to define a left equivariant moving frame $\rho: \mathrm{J}^{2} \rightarrow G$. Solving the corresponding normalization equations $v=0, v_{y}=1, v_{y y}=0$, produces the explicit moving frame formulas for the group parameters:

$$
\alpha=\frac{1}{\sqrt{u_{x}}}, \quad \beta=-\frac{u}{\sqrt{u_{x}}}, \quad \gamma=-\frac{u_{x x}}{u_{x}^{3 / 2}}, \quad \delta=\frac{u_{x}^{2}-\frac{1}{2} u u_{x x}}{u_{x}^{3 / 2}},
$$

where, for simplicity, we restrict our attention to the open subset where $u_{x}>0$. Substituting the moving frame formulae (6.13) into the higher order prolonged transformation formulae produces the normalized differential invariants. In particular, the generating differential invariant is the Schwarzian derivative

$$
\kappa=\iota\left(u_{x x x}\right)=\frac{u_{x} u_{x x x}-\frac{3}{2} u_{x x}^{2}}{u_{x}^{2}} .
$$

The transformation rules for the contact forms $\theta=d u-u_{x} d x, \theta_{x}=d u_{x}-u_{x x} d x$, etc. can be found by taking the first variation of the prolonged group transformations (6.12):

$$
\begin{aligned}
& \theta \longmapsto \frac{\theta}{(\gamma u+\delta)^{2}}, \quad \theta_{x} \longmapsto \frac{(\gamma u+\delta) \theta_{x}-2 \gamma u_{x} \theta}{(\gamma u+\delta)^{3}}, \\
& \theta_{x x} \longmapsto \frac{(\gamma u+\delta)^{2} \theta_{x x}-4 \gamma u_{x}(\gamma u+\delta) \theta_{x}-2 \gamma\left[(\gamma u+\delta) u_{x x}-2 \gamma u_{x}^{2}\right] \theta}{(\gamma u+\delta)^{4}},
\end{aligned}
$$


and so on. Substituting the moving frame formulae (6.13) produces the invariant contact forms:

$$
\begin{aligned}
& \vartheta=\iota(\theta)=\frac{\theta}{u_{x}}, \quad \vartheta_{1}=\iota\left(\theta_{x}\right)=\frac{u_{x} \theta_{x}-u_{x x} \theta}{u_{x}^{2}}, \\
& \vartheta_{2}=\iota\left(\theta_{x x}\right)=\frac{u_{x}^{2} \theta_{x x}-2 u_{x} u_{x x} \theta_{x}+\frac{7}{2} u_{x}^{2} \theta}{u_{x}^{3}},
\end{aligned}
$$

The recurrence formulae (4.6), of which the first two are

$$
\vartheta_{1}=D_{x} \vartheta=\vartheta_{x}, \quad \vartheta_{2}=D_{x}^{2} \vartheta-\kappa \vartheta=\vartheta_{x x}-\kappa \vartheta
$$

can be either deduced directly, or by applying the recurrence formulae (4.4) for invariant contact forms using the formulae for the pulled back Maurer-Cartan forms deduced below.

In view of the formula (6.15) for the order 0 invariant contact form $\vartheta$, the dual invariant normal (6.2) is $\mathfrak{n}=u_{x} \partial_{u}$, and hence the most general PSL(2)-invariant curve flow (6.1) has the form

$$
\frac{\partial u}{\partial t}=u_{x} J
$$

where $J$ is an arbitrary differential invariant. The two simplest cases occur when $J=$ 1 , leading to the trivial hyperbolic flow $u_{t}=u_{x}$, and setting $J=\kappa$, resulting in the Schwarzian KdV equation, [26],

$$
u_{t}=u_{x}^{-1}\left(u_{x} u_{x x x}-\frac{3}{2} u_{x x}^{2}\right) .
$$

To obtain the invariant Maurer-Cartan forms, we write out the recurrence formulae (4.4) for the cross-section variables $u, u_{x}, u_{x x}$ :

$$
\begin{aligned}
& 0=d \iota(u)=\iota(d u)+\nu^{1}+\iota(u) \nu^{2}+\iota\left(u^{2}\right) \nu^{3}=\varpi+\vartheta+\nu^{1}, \\
& 0=d \iota\left(u_{x}\right)=\iota\left(d u_{x}\right)+\iota\left(u_{x}\right) \nu^{2}+\iota\left(2 u u_{x}\right) \nu^{3}=\vartheta_{1}+\nu^{2}, \\
& 0=d \iota\left(u_{x x}\right)=\iota\left(d u_{x x}\right)+\iota\left(u_{x x}\right) \nu^{2}+\iota\left(2 u u_{x x}+2 u_{x}^{2}\right) \nu^{3}=\kappa \varpi+\vartheta_{2}+2 \nu^{3},
\end{aligned}
$$

with $\varpi=d x$. Solving these linear equations, and taking (6.16) into account, the pulled back Maurer-Cartan forms are

$$
\nu^{1}=-\varpi-\vartheta, \quad \nu^{2}=-\vartheta_{x}, \quad \nu^{3}=\rho^{*}\left(\mu^{3}\right)=-\frac{1}{2} \kappa \varpi-\frac{1}{2} \vartheta_{x x}-\frac{1}{2} \kappa \vartheta,
$$

from which we deduce the Maurer-Cartan invariants and Maurer-Cartan operator:

$$
\boldsymbol{\kappa}=\left(\begin{array}{c}
-1 \\
0 \\
-\frac{1}{2} \kappa
\end{array}\right), \quad \mathcal{C}=\left(\begin{array}{c}
-1 \\
-\mathcal{D} \\
-\frac{1}{2} \mathcal{D}^{2}-\frac{1}{2} \kappa
\end{array}\right)
$$

The operator (6.11) rewritten in terms of the Maurer-Cartan invariants is

$$
\mathcal{Q}=\left(\begin{array}{ccc}
\mathcal{D} & -1 & 0 \\
\kappa & \mathcal{D} & -2 \\
0 & \frac{1}{2} \kappa & \mathcal{D}
\end{array}\right)
$$


while the Poisson operator (2.14) is obtained by right multiplication by $B^{-1}$, and so

$$
\mathcal{P}=\mathcal{Q} B^{-1}=\left(\begin{array}{ccc}
0 & -2 & \mathcal{D} \\
2 & 2 \mathcal{D} & -\kappa \\
\mathcal{D} & \kappa & 0
\end{array}\right)
$$

Note that $\mathcal{P}$ is clearly skew-adjoint, but needs to be reduced in order to define a Poisson structure. Using the recurrence formulae, we discover that the invariant vertical derivatives of the Maurer-Cartan invariants are

$$
d_{\mathcal{V}}(-1)=0, \quad d_{\mathcal{V}}(0)=0, \quad d_{\mathcal{V}}(-\kappa)=-\frac{1}{2} \vartheta_{x x x}-\kappa \vartheta_{x}-\frac{1}{2} \kappa_{x} \vartheta
$$

and hence the invariant linearization operator factors as in (4.16):

$$
\mathcal{A}=\left(\begin{array}{c}
0 \\
0 \\
-\frac{1}{2}\left(\mathcal{D}^{3}+2 \kappa \mathcal{D}+\kappa_{x}\right)
\end{array}\right)=\mathcal{Q} \cdot \mathcal{C} .
$$

Notice that $\mathcal{A}$ describes the evolution of the Maurer-Cartan invariants, and so this implies that, when $u$ evolves according to the invariant curve flow (6.17), its Schwarzian invariant satisfies

$$
-\frac{1}{2} \kappa_{t}=-\frac{1}{2}\left(\mathcal{D}^{3}+2 \kappa \mathcal{D}+\kappa_{x}\right) J .
$$

In particular, when $J=\kappa$, and so we are dealing with the Schwarzian KdV flow, the induced differential invariant flow (6.20) is the ordinary Korteweg-deVries equation.

To obtain the reduced Hamiltonian and the compatibility condition, we need to find the operator $\mathcal{R}$ satisfying (5.4). In this case, the isotropy subalgebra and its annihilator have the form

$$
\mathfrak{n}=\left(\begin{array}{c}
0 \\
* \\
*
\end{array}\right), \quad \mathfrak{n}_{0}^{*}=\left(\begin{array}{c}
* \\
0 \\
0
\end{array}\right), \quad \mathfrak{n}_{0}=B^{-1} \mathfrak{n}_{0}^{*}=\left(\begin{array}{c}
0 \\
0 \\
*
\end{array}\right) .
$$

Recall that an extension $H$ will coincide with $h$ on $\boldsymbol{\kappa}$. If we want to find the reduced Hamiltonian evolution of $\kappa$ itself, then we set $\mathcal{R}=(2, X, Y)^{T}$, where the constant entry is placed in the dual position to that of the generating invariant $\kappa$ in $\boldsymbol{\kappa}$, with value 2 since the third entry of $\kappa$ is $-\frac{1}{2} \kappa$. The condition

$$
\mathcal{Q} \mathcal{R} \delta h=\mathcal{Q}\left(\begin{array}{l}
2 \\
X \\
Y
\end{array}\right) \delta h=\left(\begin{array}{c}
2 \mathcal{D}-X \\
\mathcal{D} X+2 \kappa-2 Y \\
\mathcal{D} Y+\frac{1}{2} \kappa X
\end{array}\right) \delta h=\left(\begin{array}{l}
0 \\
0 \\
*
\end{array}\right) \in \mathfrak{n}_{0}
$$

implies that $X=2 \mathcal{D}$ and $Y=\mathcal{D}^{2}+\kappa$. Thus, in this particular example, $\mathcal{R}=-2 \mathcal{C}$. We conclude that the reduced Poisson operator is given by

$$
\mathcal{P}_{R}=\mathcal{R}^{*} \cdot \mathcal{P} \cdot \mathcal{R}=-2\left(\mathcal{D}^{3}+2 \kappa \mathcal{D}+\kappa_{x}\right),
$$

which is the well-known second Hamiltonian structure of the Korteweg-deVries equation, [22]. The reduced Hamiltonian evolution of $\kappa$ is given by

$$
\kappa_{t}=\mathcal{P}_{R} \delta h=-2\left(\mathcal{D}^{3}+2 \kappa \mathcal{D}+\kappa_{x}\right) \frac{\delta h}{\delta \kappa} .
$$


Owing to the form of $\mathfrak{n}$, the compatibility condition (6.9) requires that the first entry of $\mathcal{R} \delta h$ coincides with the first entry of $\mathcal{C} J$, which requires $J=-2 \delta h$. This condition clearly makes the flows (6.20) and (6.21) coincide.

Example 6.3. Centro-equi-affine plane curves: Consider the standard linear representation of $G=\mathrm{SL}(2)$ on $M=\mathbb{R}^{2}$. The infinitesimal generators are

$$
\mathbf{v}_{1}=-u \partial_{u}+v \partial_{v}, \quad \mathbf{v}_{2}=v \partial_{u}, \quad \mathbf{v}_{3}=u \partial_{v} .
$$

The cocycle is the Killing form:

$$
\beta=2\left(\mu^{1}\right)^{2}-2 \mu^{2} \mu^{3}, \quad \text { so that } \quad B=\left(\begin{array}{lll}
2 & 0 & 0 \\
0 & 0 & 1 \\
0 & 1 & 0
\end{array}\right) .
$$

Adopting the following moving frame normalizations

$$
u \longmapsto 0, \quad v \longmapsto 1, \quad v_{x} \longmapsto 0,
$$

the generating differential invariants are

$$
\kappa=\iota\left(u_{x}\right), \quad \tau \kappa=\iota\left(v_{x x}\right) .
$$

The Maurer-Cartan invariants and operator

$$
\kappa=\left(\begin{array}{c}
0 \\
-\kappa \\
-\tau
\end{array}\right), \quad \mathcal{C}=\left(\begin{array}{cc}
0 & -1 \\
-1 & 0 \\
-\frac{\tau}{\kappa} & -\frac{1}{\kappa} \mathcal{D}
\end{array}\right)
$$

are found using the invariant variational bicomplex constructions, as in the previous example; in the interests of brevity, we suppress the details. The pulled back Poisson operator and its counterpart are

$$
\mathcal{P}=\left(\begin{array}{ccc}
2 \mathcal{D} & 2 \tau & -2 \kappa \\
-2 \tau & 0 & \mathcal{D} \\
2 \kappa & \mathcal{D} & 0
\end{array}\right), \quad \mathcal{Q}=\left(\begin{array}{ccc}
\mathcal{D} & \tau & -\kappa \\
2 \kappa & \mathcal{D} & 0 \\
-2 \tau & 0 & \mathcal{D}
\end{array}\right)
$$

Thus, in accordance with (4.16), the invariant linearization operator factorizes as

$$
\mathcal{A}=\left(\begin{array}{cc}
0 & 0 \\
-\mathcal{D} & -2 \kappa \\
-\mathcal{D} \frac{\tau}{\kappa} & -\mathcal{D} \frac{1}{\kappa} \mathcal{D}+2 \tau
\end{array}\right)=\mathcal{Q} \cdot \mathcal{C}
$$

and the Maurer-Cartan flow $\kappa_{t}=\mathcal{A}(J)$ takes the explicit form

$$
\kappa_{t}=\mathcal{D} J_{1}+2 \kappa J_{2}, \quad \tau_{t}=\mathcal{D}\left(\frac{\tau}{\kappa} J_{1}\right)+\left(\mathcal{D} \frac{1}{\kappa} \mathcal{D}-2 \tau\right) J_{2} .
$$

The isotropy subalgebra and its annihilator are

$$
\mathfrak{n}=\left(\begin{array}{c}
0 \\
0 \\
*
\end{array}\right), \quad \mathfrak{n}_{0}^{*}=\left(\begin{array}{c}
* \\
* \\
0
\end{array}\right), \quad \mathfrak{n}_{0}=B^{-1} \mathfrak{n}_{0}^{*}=\left(\begin{array}{c}
* \\
0 \\
*
\end{array}\right) .
$$


The entries dual to the position of $-\kappa$ and $-\tau$ in $\boldsymbol{\kappa}$ are third and second entries, respectively, and therefore

$$
\mathcal{R}=\left(\begin{array}{cc}
X & Y \\
0 & -1 \\
-1 & 0
\end{array}\right), \quad \mathcal{Q} \cdot \mathcal{R}=\left(\begin{array}{cc}
\mathcal{D} X-\kappa & \mathcal{D} Y-\tau \\
2 \kappa X & 2 \kappa Y-\mathcal{D} \\
-2 \tau X-\mathcal{D} & -2 \tau Y
\end{array}\right)
$$

Condition (5.4) requires $X=0, Y=(2 \kappa)^{-1} \mathcal{D}$, and thus

$$
\mathcal{R}=\left(\begin{array}{cc}
0 & \frac{1}{2 \kappa} \mathcal{D} \\
0 & -1 \\
-1 & 0
\end{array}\right), \quad \mathcal{P}_{R}=\mathcal{R}^{*} \cdot \mathcal{P} \cdot \mathcal{R}=\left(\begin{array}{cc}
0 & 0 \\
0 & -\frac{1}{2} \mathcal{D} \frac{1}{\kappa} \mathcal{D} \frac{1}{\kappa} \mathcal{D}+\mathcal{D} \frac{\tau}{\kappa}+\frac{\tau}{\kappa} \mathcal{D}
\end{array}\right)
$$

Thus, the reduced Hamiltonian flow $\boldsymbol{\kappa}_{t}=\mathcal{P}_{R} \delta h$ becomes

$$
\kappa_{t}=0, \quad \tau_{t}=\left(-\frac{1}{2} \mathcal{D} \frac{1}{\kappa} \mathcal{D} \frac{1}{\kappa} \mathcal{D}+\mathcal{D} \frac{\tau}{\kappa}+\frac{\tau}{\kappa} \mathcal{D}\right) \frac{\delta h}{\delta \tau},
$$

and so, for fixed $\kappa$, the differential invariant $\tau$ has a Hamiltonian evolution. In this example, any reduced Hamiltonian evolution will fix the value of the "arc length" invariant $\kappa$ to a nonzero constant value, while the evolution of $\tau$ inherits the second Korteweg-deVries Hamiltonian structure. Finally, since a complement to $\mathfrak{n}$ in $\mathfrak{g}$ is given by the first two entries, the compatibility condition (6.9) says

$$
J_{1}=h_{\tau}, \quad J_{2}=-\frac{1}{2 \kappa} \mathcal{D} h_{\tau}=-\frac{1}{2 \kappa} \mathcal{D} J_{1} .
$$

Example 6.4. A parabolic $\mathrm{SL}(3)$ action. Let $(u, v, w) \in M \simeq \mathbb{R}^{3}$ be identified with upper triangular $3 \times 3$ matrices with unit diagonal:

$$
U=\left(\begin{array}{ccc}
1 & u & v \\
0 & 1 & w \\
0 & 0 & 1
\end{array}\right)
$$

Consider the action of $G=\mathrm{SL}(3)$ on $M$ that corresponds to the parabolic manifold associated to the finest gradation of $\mathfrak{s l}(3)=\mathfrak{g}_{-2} \oplus \mathfrak{g}_{-1} \oplus \mathfrak{g}_{0} \oplus \mathfrak{g}_{1} \oplus \mathfrak{g}_{2}$, with

$$
\begin{array}{cc}
\mathfrak{g}_{-2}=\left(\begin{array}{ccc}
0 & 0 & * \\
0 & 0 & 0 \\
0 & 0 & 0
\end{array}\right), & \mathfrak{g}_{-1}=\left(\begin{array}{ccc}
0 & * & 0 \\
0 & 0 & * \\
0 & 0 & 0
\end{array}\right), \\
\mathfrak{g}_{1}=\left(\begin{array}{lll}
0 & 0 & 0 \\
* & 0 & 0 \\
0 & * & 0
\end{array}\right), & \mathfrak{g}_{0}=\left(\begin{array}{lll}
0 & 0 & 0 \\
0 & 0 & 0 \\
* & 0 & 0
\end{array}\right) .
\end{array}
$$

Namely, $A \in \mathrm{SL}(3)$ acts via

$$
A: U \longmapsto \widehat{U}, \quad \text { where } \quad A U=\widehat{U} C
$$


for some lower triangular unimodular matrix $C$. The infinitesimal generators of this action can be determined by standard methods:

$$
\begin{array}{lcc}
\mathbf{v}_{1}=\partial_{v}, & \mathbf{v}_{2}=\partial_{w}, & \mathbf{v}_{3}=\partial_{u}+w \partial_{v} \\
\mathbf{v}_{4}=u \partial_{u}-w \partial_{w}, & \mathbf{v}_{5}=u \partial_{u}+2 v \partial_{v}+w \partial_{w}, & \mathbf{v}_{6}=-u^{2} \partial_{u}+v \partial_{w}, \\
\mathbf{v}_{7}=(u w-v) \partial_{u}-v w \partial_{v}-w^{2} \partial_{w}, & \mathbf{v}_{8}=u(u w-v) \partial_{u}-v^{2} \partial_{v}-v w \partial_{w} .
\end{array}
$$

The cocycle is the Killing form:

$$
\beta=\frac{2}{3}\left(\mu^{4}\right)^{2}+2\left(\mu^{5}\right)^{2}+2 \mu^{1} \mu^{8}+2 \mu^{2} \mu^{7}+2 \mu^{3} \mu^{6},
$$

so that

$$
B=\left(\begin{array}{llllllll}
0 & 0 & 0 & 0 & 0 & 0 & 0 & 1 \\
0 & 0 & 0 & 0 & 0 & 0 & 1 & 0 \\
0 & 0 & 0 & 0 & 0 & 1 & 0 & 0 \\
0 & 0 & 0 & \frac{2}{3} & 0 & 0 & 0 & 0 \\
0 & 0 & 0 & 0 & 2 & 0 & 0 & 0 \\
0 & 0 & 1 & 0 & 0 & 0 & 0 & 0 \\
0 & 1 & 0 & 0 & 0 & 0 & 0 & 0 \\
1 & 0 & 0 & 0 & 0 & 0 & 0 & 0
\end{array}\right) .
$$

The prolonged action is locally free on a dense open subset of $\mathrm{J}^{2}$. We choose a crosssection $K^{2} \subset \mathrm{J}^{2}$ that specifies the following moving frame normalizations:

$$
u \rightarrow 0, \quad v \rightarrow 0, \quad w \rightarrow 0, \quad u_{x} \rightarrow 0, \quad v_{x} \rightarrow 1, \quad w_{x} \rightarrow 0, \quad u_{x x} \rightarrow 1, \quad v_{x x} \rightarrow 0 .
$$

The resulting generating differential invariants are

$$
\kappa=\iota\left(w_{x x}\right), \quad \tau=\iota\left(u_{x x x}\right), \quad \rho=\iota\left(v_{x x x}\right) .
$$

Using the invariant variational bicomplex construction, the Maurer-Cartan invariants and operator are found to be

$$
\boldsymbol{\kappa}=\left(\begin{array}{c}
-1 \\
0 \\
0 \\
-\tau \\
0 \\
-\kappa \\
1 \\
\frac{1}{2} \rho
\end{array}\right), \quad \mathcal{C}=\left(\begin{array}{ccc}
0 & -1 & 0 \\
0 & 0 & -1 \\
-1 & 0 & 0 \\
-\mathcal{D}^{2}-2 \tau \mathcal{D}-\tau_{x}-\tau^{2}-\frac{1}{2} \rho & -\frac{3}{2} \mathcal{D}-\tau & 0 \\
0 & -\frac{1}{2} \mathcal{D} & 0 \\
0 & -\kappa & -\mathcal{D}+\tau \\
\mathcal{D}+\tau & 1 & 0 \\
-\frac{1}{2} \kappa & \frac{1}{2} \mathcal{D}^{2}+\frac{1}{2} \rho & \frac{1}{2}
\end{array}\right)
$$


On $\mathcal{L} \mathfrak{g}^{*}$, the Poisson operator is

$$
\mathcal{P}=\left(\begin{array}{cccccccc}
0 & 0 & 0 & 0 & -\rho & -1 & -\kappa & \mathcal{D} \\
0 & 0 & -\frac{1}{2} \rho & 1 & -1 & 0 & \mathcal{D}+\tau & 0 \\
0 & \frac{1}{2} \rho & 0 & \kappa & \kappa & \mathcal{D}-\tau & 0 & 0 \\
0 & -1 & -\kappa & \frac{2}{3} \mathcal{D} & 0 & 0 & 0 & 0 \\
\rho & 1 & -\kappa & 0 & 2 \mathcal{D} & 0 & 0 & 2 \\
1 & 0 & \mathcal{D}+\tau & 0 & 0 & 0 & 1 & 0 \\
\kappa & \mathcal{D}-\tau & 0 & 0 & 0 & -1 & 0 & 0 \\
\mathcal{D} & 0 & 0 & 0 & -2 & 0 & 0 & 0
\end{array}\right)
$$

whereas

$$
\mathcal{Q}=\left(\begin{array}{cccccccc}
\mathcal{D} & 0 & 0 & 0 & -2 & 0 & 0 & 0 \\
\kappa & \mathcal{D}-\tau & 0 & 0 & 0 & -1 & 0 & 0 \\
1 & 0 & \mathcal{D}+\tau & 0 & 0 & 0 & 1 & 0 \\
0 & -\frac{3}{2} & -\frac{3}{2} \kappa & \mathcal{D} & 0 & 0 & 0 & 0 \\
\frac{1}{2} \rho & \frac{1}{2} & -\frac{1}{2} \kappa & 0 & \mathcal{D} & 0 & 0 & 1 \\
0 & \frac{1}{2} \rho & 0 & \kappa & \kappa & \mathcal{D}-\tau & 0 & 0 \\
0 & 0 & -\frac{1}{2} \rho & 1 & -1 & 0 & \mathcal{D}+\tau & 0 \\
0 & 0 & 0 & 0 & -\rho & -1 & -\kappa & \mathcal{D}
\end{array}\right) .
$$

The invariant linearization operator $\mathcal{A}=\mathcal{Q} \cdot \mathcal{C}$ is

$$
\left(\begin{array}{ccc}
0 & 0 & 0 \\
0 & 0 & 0 \\
0 & 0 & 0 \\
-\mathcal{D}^{3}-2 \tau \mathcal{D}^{2}-\left(3 \tau_{x}+\frac{1}{2} \rho+\tau^{2}\right) \mathcal{D}- & -\frac{3}{2} \mathcal{D}^{2}-\tau \mathcal{D}-\tau_{x} & \frac{3}{2} \\
-\tau_{x x}-2 \tau \tau_{x}-\frac{1}{2} \rho_{x}+\frac{3}{2} \kappa & 0 & 0 \\
0 & 0 & 0 \\
-\kappa \mathcal{D}^{2}-2 \kappa \tau \mathcal{D}-\kappa \tau_{x}-\kappa \tau^{2}-\frac{1}{2} \kappa \rho & -3 \kappa \mathcal{D}-\kappa_{x} & -\mathcal{D}^{2}+2 \tau \mathcal{D}+\tau_{x}-\tau^{2}-\frac{1}{2} \rho \\
0 & 0 & \frac{3}{2} \mathcal{D}-\tau
\end{array}\right)
$$


Next, the isotropy subalgebra and annihilator are

$$
\mathfrak{n}=\left(\begin{array}{c}
0 \\
0 \\
0 \\
* \\
* \\
* \\
* \\
*
\end{array}\right), \quad \mathfrak{n}_{0}^{*}=\left(\begin{array}{c}
* \\
* \\
* \\
0 \\
0 \\
0 \\
0 \\
0
\end{array}\right), \quad \mathfrak{n}_{0}=B \mathfrak{n}_{0}^{*}=\left(\begin{array}{c}
0 \\
0 \\
0 \\
0 \\
0 \\
* \\
* \\
*
\end{array}\right) .
$$

If

$$
\mathcal{R}=\left(\begin{array}{ccc}
0 & 0 & 2 \\
X_{2} & Y_{2} & Z_{2} \\
-1 & 0 & 0 \\
0 & -\frac{3}{2} & 0 \\
X_{5} & Y_{5} & Z_{5} \\
X_{6} & Y_{6} & Z_{6} \\
X_{7} & Y_{7} & Z_{7} \\
X_{8} & Y_{8} & Z_{8}
\end{array}\right)
$$

then

$$
\mathcal{Q} \mathcal{R}=\left(\begin{array}{ccc}
-2 X_{5} & -2 Y_{5} & -2 Z_{5}+2 \mathcal{D} \\
(\mathcal{D}-\tau) X_{2}-X_{6} & (\mathcal{D}-\tau) Y_{2}-Y_{6} & (\mathcal{D}-\tau) Z_{2}-Z_{6}+2 \kappa \\
-(\mathcal{D}+\tau)+X_{7} & Y_{7} & Z_{7}+2 \\
-\frac{3}{2} X_{2}+\frac{3}{2} \kappa & -\frac{3}{2} Y_{2}-\frac{3}{2} \mathcal{D} & -\frac{3}{2} Z_{2} \\
\frac{1}{2} X_{2}+\frac{1}{2} \kappa+\mathcal{D} X_{5}+X_{8} & \frac{1}{2} Y_{2}+\mathcal{D} Y_{5}+Y_{8} & \frac{1}{2} Z_{2}+\rho+\mathcal{D} Z_{5}+Z_{8} \\
* & * & * \\
* & * & * \\
* & * & *
\end{array}\right)
$$

and so condition $(5.4)$ results in

$$
\mathcal{R}=\left(\begin{array}{ccc}
0 & 0 & 2 \\
\kappa & -\mathcal{D} & 0 \\
-1 & 0 & 0 \\
0 & -\frac{3}{2} & 0 \\
0 & 0 & \mathcal{D} \\
(\mathcal{D}-\tau) \kappa & -(\mathcal{D}-\tau) \mathcal{D} & 2 \kappa \\
\mathcal{D}+\tau & 0 & -2 \\
-\kappa & \frac{1}{2} \mathcal{D} & -\mathcal{D}^{2}-\rho
\end{array}\right)
$$

From here, the reduced Poisson operator $\mathcal{P}_{R}$ is given by

$$
\left(\begin{array}{ccc}
\kappa(\mathcal{D}+\tau)^{2}-(\mathcal{D}-\tau)^{2} \kappa & \frac{1}{2} \rho \mathcal{D}+(\mathcal{D}-\tau)^{2} \mathcal{D} & -2(\kappa \mathcal{D}+(\mathcal{D}-\tau) \kappa+\kappa(\mathcal{D}+\tau)) \\
\frac{1}{2} \mathcal{D} \rho+\mathcal{D}(\mathcal{D}+\tau)^{2} & -\frac{3}{2} \mathcal{D} & -3 \mathcal{D}^{2}-2 \mathcal{D} \tau \\
-2(\mathcal{D} \kappa+\kappa(\mathcal{D}+\tau)+(\mathcal{D}-\tau) \kappa) & 3 \mathcal{D}^{2}-2 \tau \mathcal{D} & -2\left(\mathcal{D}^{3}+\mathcal{D} \rho+\rho \mathcal{D}\right)
\end{array}\right)
$$


Finally, the compatibility condition (6.9) is given by equating the portions of $\mathcal{R} \delta h$ and $\mathcal{C} J$ that correspond to a complement of $\mathfrak{n}$ in $\mathfrak{g}$; that is, by equating the first 3 entries of each. This results in

$$
-\left(\begin{array}{c}
J_{2} \\
J_{3} \\
J_{1}
\end{array}\right)=\left(\begin{array}{c}
2 h_{\rho} \\
\kappa h_{\kappa}-\left(h_{\tau}\right)_{x} \\
-h_{\kappa}
\end{array}\right),
$$

where the subscripts denote variational derivatives.

Acknowledgments: We would like to thank the referee for helping us to improve the paper.

\section{References}

[1] Chou, K.-S., and Qu, C., Integrable equations arising from motions of plane curves, Physica D 162 (2002), 9-33.

[2] Chou, K.-S., and Qu, C.-Z., Integrable equations arising from motions of plane curves II, J. Nonlinear Sci. 13 (2003), 487-517.

[3] Doliwa, A., and Santini, P.M., An elementary geometric characterization of the integrable motions of a curve, Phys. Lett. A 185 (1994), 373-384.

[4] Dubrovin, B.A., and Novikov, S.P., On Poisson brackets of hydrodynamic type, Sov. Math. Dokl. 30 (1984), 651-654.

[5] Dubrovin, B.A., and Novikov, S.P., Hydrodynamics of weakly deformed soliton lattices. Differential geometry and Hamiltonian theory, Russian Math. Surveys 44:6 (1989), 35-124.

[6] Fels, M., and Olver, P.J., Moving coframes. II. Regularization and theoretical foundations, Acta Appl. Math. 55 (1999), 127-208.

[7] Green, M.L., The moving frame, differential invariants and rigidity theorems for curves in homogeneous spaces, Duke Math. J. 45 (1978), 735-779.

[8] Guggenheimer, H.W., Differential Geometry, McGraw-Hill, New York, 1963.

[9] Hasimoto, H., A soliton on a vortex filament, J. Fluid Mech. 51 (1972), 477-485.

[10] Hubert, E., Differential invariants of a Lie group action: syzygies on a generating set, J. Symb. Comp. 44 (2009), 382-416.

[11] Ivey, T., Integrable geometric evolution equations for curves, Contemp. Math. 285 (2001), 71-84.

[12] Kirillov, A.A., Merits and demerits of the orbit method, Bull. Amer. Math. Soc. 36 (1999), 433-488.

[13] Kogan, I.A., and Olver, P.J., Invariant Euler-Lagrange equations and the invariant variational bicomplex, Acta Appl. Math. 76 (2003), 137-193.

[14] Mansfield, E.L., and van der Kamp, P.E., Evolution of curvature invariants and lifting integrability, J. Geom. Phys. 56 (2006), 1294-1325.

[15] Marí Beffa, G., Poisson brackets associated to the conformal geometry of curves, Trans. Amer. Math. Soc. 357 (2005), 2799-2827. 
[16] Marí Beffa, G., Poisson geometry of differential invariants of curves in some nonsemisimple homogeneous spaces, Proc. Amer. Math. Soc. 134 (2006), 779-791.

[17] Marí Beffa, G., Hamiltonian structures on the space of differential invariants of curves in flat semisimple homogenous manifolds, Asian J. Math. 12 (2008), $1-33$.

[18] Marí Beffa, G., On bi-Hamiltonian flows and their realizations as curves in real semisimple homogeneous manifolds, Pacific J. Math., to appear.

[19] Marí Beffa, G., Sanders, J.A., and Wang, J.P., Integrable systems in threedimensional Riemannian geometry, J. Nonlinear Sci. 12 (2002), 143-167.

[20] Marsden, J.E., and Ratiu, T., Reduction of Poisson manifolds, Lett. Math. Phys. 11 (1986), 161-169.

[21] Nakayama, K., Segur, H., and Wadati, M., Integrability and motion of curves, Phys. Rev. Lett. 69 (1992), 2603-2606.

[22] Olver, P.J., Applications of Lie Groups to Differential Equations, Second Edition, Graduate Texts in Mathematics, vol. 107, Springer-Verlag, New York, 1993.

[23] Olver, P.J., Generating differential invariants, J. Math. Anal. Appl. 333 (2007), 450-471.

[24] Olver, P.J., Invariant submanifold flows, J. Phys. A 41 (2008), 344017.

[25] Olver, P.J., Differential invariant algebras, Contemp. Math., to appear.

[26] Weiss, J., The Painlevé property for partial differential equations. II. Bäcklund transformation, Lax pairs, and the Schwarzian derivative, J. Math. Phys. 24 (1983), 1405-1413. 